\title{
Las preguntas y la ciencia escolar: una experiencia con la segunda infancia
}

The questions and the school science: An experience with the second childhood

\author{
Sandra Patricia Rojas Rojas
}

Profesora Colegio Distrital "Divino Maestro" Integrante Equipo de calidad, SED. Tutora FESAD. Universidad Pedagógica y Tecnológica de Colombia UPTC. Grupo IREC. UPN y SIEK Fesad, UPTC. Bogotá. Colombia. rojaspatty20@hotmail.com

\section{Resumen}

Se presenta una experiencia investigativa cualitativa con niños y niñas bogotanas de Educación Básica Primaria donde se pretendió recuperar la relación del preguntar en el marco de la ciencia escolar al considerarse como estrategia de enseñanza por un parte y por otra permite la construcción o reconstrucción de modelos explicativos de una disciplina, objeto de trabajo en el aula o establecer relaciones que acerquen a la comprensión de estos.

Los resultados obtenidos señalan que al favorecer, generar y fortalecer las preguntas no sólo se mantuvo un interés por estas; también en sus construcciones se evidencia que los modelos explicativos de las disciplinas en cuestión existe aproximación al lenguaje científico, a la actividad cientúfica de donde se admite la comprensión del trabajo cient́fico; en concordancia con Ferreira y De Longhi, (2001) es viable para la organización de ideas, interpretación de hallazgos, retomar ideas iniciales y superación de aprendizajes tradicionalistas.

\section{Palabras clave}

Didáctica de las ciencias, segunda infancia, estrategias didácticas, preguntas, ciencia escolar.

\section{Abstract}

A qualitative investigative experience is presented with children and girls from Bogotá of Primary Basic Education where he/she sought to recover the relationship of asking in the mark from the school science when being considered as teaching strategy for an it leaves and for another it allows the construction or reconstruction of explanatory models of a discipline, work object in the dassroom or relationships that bring near to the understanding of these to settle down.

The obtained results point out that when favoring, to generate and to strengthen the questions not only stayed an interest for these; also in their constructions it is evidenced that the explanatory models of the disciplines in question approach exists to the scientific language, to the scientific activity of where the understanding of the scientific work is admitted; in agreement with Barbosa, de Carvalho, Ferreira y de Longhi, (2001) it is viable for the organization of ideas, interpretation of discoveries, to recapture initial ideas and separation of traditionalist leamings.

\section{Keywords}

Didactics of the sciences, second childhood, didactic strategies, you ask, school science. 


\section{Introducción}

Hoy en día en los contextos educativos poco se habla y se hace uso de los legados de Sócrates y mucho menos el continuado por su disćpulo "Platón" presentado en sus "diálogos"; pareciera que en el aula, el docente es aquel que realiza las preguntas a sus dicentes muy pocas o nunca lo contrario, situación que obliga a pensar en una respuesta inmediata y buscando tal vez satisfacerlo (a) al docente, tímidamente las plantean o terminan aceptando respuestas inadecuadas sin que se generen espacios de reflexión, pareciera que se le impidiera que resuelvan y propongan situaciones adversas ¿Es esta la finalidad de una enseñanza de las ciencias? y clo es la pregunta? "lo importante es que la pregunta intrigue, cuestione al niño o niña y lo motive a reasignar significados o como lo afirma Kamil (1983, citado por Eslava y Eslava, 2000) "a construir conocimiento al poner las cosas dentro de relaciones coherentes"

Dada las intenciones preliminares ¿Qué sucede al interior de nuestras aulas? ¿Es posible que los anteriores cuestionamientos sean los causantes de aulas donde imperen las respuestas? ¿Cómo recobrar el sentido de preguntar en las y los estudiantes? Cómo y para qué son necesarias las preguntas en la comprensión y explicación que demanda la ciencia escolar? en el proceso enseñanza-aprendizaje? y al emplearla ¿se propende hacia el aprendizaje significativo? ¿Por qué el niño o la niña poco o nada se cuestiona sobre hechos, fenómenos, situaciones naturales? ¿Existen los espacios académicos en el que se promueva las preguntas de calidad? ¿Estamos los docentes formulando preguntas de calidad? Podría pensarse que ¿Nos encontramos inmersos en el paradigma transmisión repetición? ¿Sólo lo inmediato y cercano a nuestros sentidos es lo aceptado? ¿Estaríamos centrados al momento de enseñar conceptos en ciencias desde versiones empiropositivstas? ¿o es el docente quién presenta dificultades en la formulación de preguntas de calidad? A respecto Eslava y Eslava (2002) señala que el maestro desconoce el arte de preguntar en el aula con calidad y si lo hace tiende a enfatizar aquellas con tendencia memorística, desauida las que invitan a la síntesis y la evaluación; y continúa afirmando que las preguntas de calidad además de hacer amena la dase, promueve el desarrollo del pensamiento lógico y de la expresión verbal, "lo importante es que la pregunta intrigue, cuestione al niño o niña y lo motive a reasignar significados o como lo describe Kamil (1983, citado por Eslava y Eslava, 2000) "a construir conocimiento al poner las cosas dentro de relaciones coherentes"

\section{Consideraciones conceptuales}

\section{¿Y......por qué y para qué preguntar en ciencias?}

En la actualidad, la didáctica de las ciencias es una disciplina teóricamente fundamentada (Gil y Colab. 1999; 2002 citado por Pérez, Gallego, Torres de Gallego y Gallego, 2003) que posee unos campos de conocimiento e investigación (Gil, Carrascosa y Martínez Terrades, 1999) y en consecuencia "es una ciencia de enseñar ciencias" (Sanmartí, 2001). Presentado de este modo, los didactas de las ciencias experimentales se enfrentan a la formulación y solución de tres problemas espećíicos: el de la enseñabilidad, el de la enseñanza y el de las relaciones entre enseñanza y aprendizaje (Pérez, Gallego, Torres de Gallego y Gallego, 2003); esta investigación se enmarca dentro del segundo en el que, estrategias didácticas como "la pregunta" contribuye a la solución de esta problemática.

La revisión bibliográfica acerca del problema que nos compete, permitió confrontar y analizar los reportes de diferentes investigaciones alrededor de esta estrategia didáctica que respondería en buena parte la cuestión inicial, al establecer su papel en la enseñanza de las ciencias y contextualizada desde la didáctica cientúfica más no como práctica instrumental llanamente. Al trazársela en esta perspectiva, el didacta concreta la formulación metodológica en términos de estrategias de enseñanza que supone involucrarán y comprometerían a los y las estudiantes en el estudio de cada unidad temática (Pérez, Gallego, Torres de Gallego y Gallego, 2003) 
Al respecto, emergen discusiones al pensarse la pregunta como un canal para la interpretación en nuevas situaciones, el reconocimiento al considerarse como vehículo de acercamiento a la actividad científica (Sutton, 2003); poner aprueba ideas, conjeturar hipótesis, crear significados y visiones del mundo (Tamayo y Sanmarti, 2001) y comunicar y expresar conceptos (Maćas y Maturano, 2001) reconstruidos o construidos desde la ciencia objeto de trabajo en el aula. En esta misma vía, se rescata investigaciones como la de, Rojas, Vargas y Castellanos (2002) quienes puntualizan en que "las preguntas" además de orientar el proceso lector, direccionan la calidad y precisión de las argumentaciones favoreciendo reconstrucción y construcción de significados y formas de significar, así como la intencionalidad comunicativa oral y escrita.

Paralelamente, Eslava y Eslava, E. (2002) al referenciar su uso oral y escrito de las preguntas, manifiesta la necesidad de retomar el arte de preguntar por los profesores para convertirla en una verdadera mediación pedagógica y que como tal, promueva a las niñas y los niños para que se pregunte y se pregunte en clase de ciencias. A su vez, Montenegro, A., (1997; 1999; 2000) ha indagado sobre la función pedagógica de la pregunta en los procesos de aprendizaje, razonamiento, influencia cognitiva y metacognitivas en Ciencias. Los resultados de estas investigaciones reflejan, que las preguntas contribuyen de manera sistemática a la construcción conceptual en los estudiantes y procesos de razonamiento en ciencia.

Caldeira, Gómez, Morgado y Otero (2001) reiteran la función de las preguntas como controladores en la comprensión de la ciencia. En la Educación Básica y Media los niños y las niñas son capaces de formularlas sobre contenidos científicos, arando tienen la oportunidad de hacerlo.

Entonces en el marco de la ciencia escolar definida como "un proceso de construcción similar al de la ciencia pero en el que las preguntas pueden ser distintas, ya que han de tener sentido para los alumnos" (Izquierdo, 1999; citado por Roca, T., Montserrat y Marquez, C., 2005) cobra mayor relevancia desde la reconstrucción que permita esta estrategia en esos modelos explicativos que los y las niñas hagan de conceptos científicos.

\section{La pregunta y la ciencia}

Acerca de lo epistemológico, filosófico y antropológico de la pregunta

Desde un aspecto filosófico y antropológico recordemos que desde la Grecia Clásica, Sócrates creó así un método denominado mayéutica (o arte de "alumbrar" los espíritus) por el que lograba que sus interlocutores descubrieran la verdad a partir de ellos mismos mediante preguntas, respuestas y más preguntas y continuado por su disápulo Platón. Hoy en día se conoce como el arte de preguntar, donde se logra llevar y llegar a una auténtica conversación, como forma de comunicación interpersonal y de responder. La pregunta tiene sentido en cuanto ella es el eje de comunicación y por lo mismo es uno de los modos universales de estar con otro (Pasquall, 1990citado por Eslava y Eslava, 2000).

En el nivel epistemológico Hans-George Gadamer (1994), en su libro Verdad y Método nos ilustra lo concemiente a la pregunta al explicar su naturaleza desde la hermenéutica dentro del análisis de la conciencia de la historia efectual. Para ello Gadamer hace una reflexión sobre la conceptualización de ésta, al referirse como el abrir la posibilidad al conocimiento con cierto sentido de orientación (la apertura de lo preguntado consiste en que no está fijada a la respuesta).

Kuhn (1971), plantea como el individuo al formularse preguntas y desde luego la misma comunidad cient́fica, las respuestas a estas son determinantes en el desarrollo científico. Bajo esta afirmación cabe aludir que a través de la historia de las ciencias, el hombre de ciencia ha avanzado y mudado a nuevas construcciones y reconstucciones de conceptos gracias al arte de preguntarse.

En consecuencia, Gadamer establece condiciones para que las preguntas conserven su identidad como tal. Una de ellas es el sentido que consiste precisamente en dejar al descubierto la auestionabilidad de lo que se pregunta sin que ocurra una "perdida de orientación" de lo 
preguntado con disponibilidad a abrirse. La verdadera pregunta requiere de esa apertura, y cuando falta no es en el fondo más que una pregunta aparente que no tiene sentido real de la pregunta, es decir, su falta de orientación no le permiten dar respuesta; otra es su horizonte, afirma que "una pregunta sin horizonte es una pregunta en vacío" lo adquiere cuando se encuentra objetividad en lo preguntado y la última es el planteamiento como consecuencia de la anterior, es decir, preguntar es pensar y se realizan dentro de un contexto determinado.

\section{El enfoque lingüístico de la pregunta}

En el caso de esta investigación se retomó y consideró la definición que asume Eslava y Eslava, (2002) desde una óptica lingüística como una proposición con sentido interrogativo que se explica por medio de signos de interrogación (c...?) al comienzo y al final de la oración. Con la pregunta expresamos inquietudes, dudas a resolver, reforzamos los nuevos significados de los o el concepto trabajado en el aula.

Por consiguiente, una pregunta de calidad lingüísticamente ha de formularse bajo las siguientes condiciones (Eslava y Eslava, 2002): comienza por un pronombre o adverbio interrogativo, que según Kant genera respuestas divergentes (lo que llamaremos a entender de los y las niñas el uso de palabras daves como: qué, para qué, dónde, quién, por qué, cuál, cómo y cuándo; se formula con oraciones compuestas (entendidas para los niños como su estructura gramatical: sujeto, predicado y verbo) escrita en signos de interrogación, se pronuncie con entonación interrogativa.

Acudiendo a las características de una pregunta de calidad (Eslava y Eslava, (2002) desde su planteamiento, horizonte y sentido son:

- $\quad$ Reflexiva: intrigante, reflexiva, crítica.

- Contextualizada: adecuada a un tiempo, a un espacio, a un tema, a una lectura.

- Decisoria: espontánea, ayuda a la toma de decisiones.

- Rećproca: bidireccional, entabla el diálogo, con sentido significativo.

- $\quad$ Divergente: abierta a múltiples respuestas.

- Lingüista: descrito anteriormente.

\section{El marco metodológico}

La hipótesis de esta investigación fue en buena parte indagar sobre las causas que originan la cultura de respuestas en las aulas; si las preguntas formuladas a partir de las unidades didácticas propuestas para abordar el concepto gases con niños y niñas de grado cuarto de Educación Básica primaria, son de calidad tal como se inscribe en las consideraciones conceptúlales anteriores.

Metodológicamente se consideraron tres fases; Para la primera, se indagó con la población objeto de estudio sobre las implicaciones y funcionalidad de la pregunta en los procesos de enseñanza -aprendizaje de las ciencias mediante un cuestionario con dos preguntas abiertas: Para ti ¿qué es preguntar? ¿Cuándo preguntas? ¿Por qué y para qué preguntas? ¿te agrada preguntar?

En la segunda "Livia de Preguntas" se optó por indagar y caracterizar en los y las dicentes la formulación (planteamiento), coherencia, sentido, claridad, calidad y estructura lingüística de las preguntas siendo estas las finalidades que orientaron la unidad didáctica No. 1; con tal propósito se realizó la lectura de un texto narrativo titulado "El Gordo Manatí de Humbolt" y su imagen representada en un acetato siendo esta actividad un instrumento de recolección.

Una vez evaluado esta fase valiéndose de la rejilla de evaluación (ver anexo No. 1) entendidas como matrices o esquemas que a manera de pantalla, permite registrar y visualizar las regularidades en los desempeños de los estudiantes frente a unos descriptores (aspectos evaluativos). El conjunto de estos datos y juicios valorativos permite inferir el final de un proceso, 
si se han comprendido los conceptos relevantes referidos (Rosas, 2002), la segunda "Mis preguntas son importantes"divisada desde dos momentos; uno de ellos fue la generación de un ambiente apropiado para la formulación de preguntas en la dase de ciencias divisados por: establecimiento de compromisos de los actores participantes (docentes-niños, niñas y viceversa) bajo criterios de tolerancia, respeto por la diferencia de pensamiento, libertad de expresión, toma de decisiones y demarcada por el lenguaje que ese emplearía para ello, sumado a esto la premisa "toda pregunta es válida", seguidamente orientar su construcción oral y escrita desde una óptica lingüística constituyéndose como instrumentos de recolección de información.

Para su formulación se desarrollaron orientaron desde unidades didácticas tituladas ¿Cómo formular preguntas? Construyo preguntas interesantes dadas desde las daves y secretos para su formulación.

\section{Claves: auándo \\ Empleo de los signos de interrogación al principio y final de la pregunta \\ Toda pregunta debe tener sujeto, predicado y verbo \\ Consejos: \\ observo muy bien lo que quiero preguntar \\ Mi pregunta debe estar relacionada con lo que quiero saber \\ Mi pregunta debe tener sentido, es decir, que quien la lea, la entienda}

Empleo de los ocho honestos servidores: cómo, qué, auál, quién, dónde, por qué, para qué y

Caldeira, Gómez, Morgado y Otero (2001) reiteran que las y los estudiantes son capaces de formular preguntas sobre contenidos científicos, cuando tienen la oportunidad de hacerlo. Rojas, Castellanos, y Vargas, (2002) conduyen que es necesario que los niños y las niñas expresen sus preguntas en razón a que al no generarse estos espacios, se propicia una cultura de la respuestas.

El otro, implica el planteamiento y desarrollo de preguntas orales y escritas al inicio, durante y final de las unidades didácticas así como los laboratorios como acercamiento al trabajo experimental referidos a los conceptos de ciencias trabajados en el aula durante el año escolar, en el que se buscó su estrecha relación entre el tema propuesto, la coherencia de las hipótesis, las interpretaciones a las observaciones y deducciones generadas con las preguntas de partida de los niños y niñas, tomándose como a la vez como instrumentos de recolección de información, evaluados en la rejilla de evaluación; Desde la perspectiva deductivista, el trabajo experimental ha de tener un sentido contrastativo entre las hipótesis de los estudiantes y del profesor de tal manera que los resultados le permitan construir y reconstruir las competencias significados en la enseñanza del concepto objeto de trabajo en el aula. Dumon (1992), afirma que el trabajo en el laboratorio debe pretender en los educandos: identificar el problema, plantear cuestionamientos y responderlos desde el lenguaje metodológico y conceptual, formular hipótesis, proponer contrastaciones experimentales de las hipótesis, poner en tela de juicio sus representaciones que ha elaborado, interpretar los resultados hallados, entre otros.

Se hizo necesario que para esta fase contemplar la Entrevista no estructurada (Briones, 2004) a los niños y las niñas como instrumento de recolección de información en razón a que se no se utilizó un sistema de preguntas propiamente dicho, sino temas centrales del objeto de estudio, sobre posiciones de los entrevistados registrada en un diario de la investigadora. 


\section{Resultados, análisis y discusión}

Al iniciar el proceso. I fase. Lluvia de Preguntas

Al indagar sobre su formulación, coherencia, seauencia, claridad, calidad y sentido, se encontró una marcada pobreza tanto en su número, como su planteamiento (una o dos), constante incoherencia entre lo leído y el concepto trabajado, resistencia a su elaboración escrita predominando la tendencia a escribir proposiciones u oraciones incompletas; es de anotarse que solían esperar las preguntas del docente para luego formular las suyas un tanto parecidas, muy pocos (4 estudiantes) deliberadamente. Obsérvese a continuación ejemplos de estas.

$>$ ¿Quién descubrió?

$>$ ¿En dónde se encuentra?

$>$ ¿Los gases producen a la gente?

$>$ ¿El gas sale de un?

$>$ ¿Por qué no se pueden ver?

$>$ ¿Qué el aire es bueno y a veces peligroso?

$>$ ¿El manatci es?

$>$ ¿Por qué vive y tiene ojos?

También se destaca su timidez al formularlas ya que consideran que pueden ser ridiculizados ante sus compañeros por dejar entre ver sus posibles interpretaciones y reconstrucciones de un texto o evento, hecho que concuerda con los resultados encontrados por Rojas, Castellanos, y Vargas, (2002). Por tanto, es necesario mantener estos espacios, de lo contrario se continúa con la cultura de las respuestas.

Durante el proceso-II fase. "Mis preguntas son importantes"

Primer momento. De la generación de un ambiente propicio para la formulación de preguntas.

Una vez concebido el clima apropiado para el preguntar mediante interpretación de lecturas, imágenes y otros, se orientó la formulación y elaboración de preguntas de calidad acudiendo a sus características (Eslava y Eslava, 2002).

Reflexiva: intrigante, reflexiva, crítica.

$>$ Contextualizada: adecuada a un tiempo, a un espacio, a un tema, a una lectura.

$>$ Decisoria: espontánea, ayuda a la toma de decisiones.

> Recíproca: bidireccional, entabla el diálogo, con sentido significativo.

Divergente: abierta a múltiples respuestas.

> Lingüista: comienza por un pronombre o adverbio interrogativo, que según Kant genera respuestas divergentes (lo que llamaremos a entender de los y las niñas el uso de palabras claves como: qué, para qué, dónde, quién, por qué, auál, cómo y auándo; se formula con oraciones compuestas (entendidas para los niños como su estructura gramatical: sujeto, predicado y verbo) escrita en signos de interrogación, se pronuncie con entonación interrogativa.

Segundo momento. Planteamiento y desarrollo de preguntas orales y escritas al inicio, durante y final de las unidades didácticas y laboratorios (trabajo experimental).

De las unidades didácticas. Los resultados que señalan la rejilla de evaluación para este instrumento indican que en el transcurso del año escolar, los niños y niñas incorporan y formulan preguntas de calidad científica al solicitarlas 0 al indagar en procesos de carácter investigativo caracterizadas por su espontaneidad, confianza y seguridad. Lama la atención que las preguntas planteadas al finalizar las unidades didácticas daban cuenta, en su gran mayoría, de sus intereses a 
favor de profundizar las temáticas emprendidas en dase, las restantes reflejaban sus dudas o vacíos conceptuales, hecho que en buen parte permitió al docente direccionar las temáticas a abordar y al estudiante, como lo afirma Gadamer (1994), reflexionar sobre lo planteado.

Obsérvese a continuación, algunas de las preguntas formuladas por los y las estudiantes en el transcurso de la aplicación de las unidades didácticas en las que se visualizan desde una perspectiva cientúfica. Es pertinente anotar que inicialmente se realizó una dasificación de éstas a partir los criterios de análisis del concepto objeto de trabajo en el aula (en este caso "gases") para los grados cuarto y tercero.

\begin{tabular}{|c|c|c|c|}
\hline \multicolumn{4}{|c|}{ LOS GASES } \\
\hline $\begin{array}{l}\text { CATIEORIAS } \\
\text { CONCEPTO }\end{array}$ & $\begin{array}{l}\text { CONCEPTO- } \\
\text { GENERALTDAES }\end{array}$ & ESTRUCTURA & $\begin{array}{l}\text { CARACTERÍSTICAS } \\
\text { GENERALES Y } \\
\text { PROPIEDADES. }\end{array}$ \\
\hline $\begin{array}{l}\text { GRADOS DE } \\
\text { ENSET̃ANZA }\end{array}$ & & & \\
\hline TERCERO & $\begin{array}{l}\text { ¿Existen los gases? } \\
\text { ¿Por qué al destapar } \\
\text { un perfume huele? } \\
\text { ¿Qué son los gases? }\end{array}$ & $\begin{array}{l}\text { ¿Qué contiene un gas por dentro? } \\
\text { ¿Por qué los gases pareciera que no } \\
\text { los vemos? } \\
\text { ¿Qué es el oxígeno? }\end{array}$ & $\begin{array}{l}\text { ¿Por qué huele el gas del } \\
\text { cilindro? } \\
\text { El gas ¿pesa? }\end{array}$ \\
\hline CUARTO & $\begin{array}{l}\text { ¿Qué clases de gases } \\
\text { hay? } \\
\text { ¿Cuáles son los gases } \\
\text { que más se utiliza? } \\
\text { ¿Qué es un gas? } \\
\text { ¿Cómo fue el primer } \\
\text { gas? ¿Quién descubrió } \\
\text { el primer gas? }\end{array}$ & $\begin{array}{l}\text { ¿Qué contiene un gas? } \\
\text { ¿Por qué el gas no se bebe? } \\
\text { ¿Cuáles son los gases nobles? } \\
\text { ¿De qué está formado un gas } \\
\text { lacrimógeno? } \\
\text { ¿Qué sustancias químicas contiene } \\
\text { un gas? } \\
\text { Los gases, ¿tienen moléculas? } \\
\text { ¿Cuántos siglos han pasado desde } \\
\text { qué descubrieron el primer gas? } \\
\text { El aire ¿es una molécula? }\end{array}$ & $\begin{array}{l}\text { ¿Por qué los gases producen } \\
\text { daño a la gente? } \\
\text { ¿Cómo se reproduce un gas? } \\
\text { ¿Los gases salen de las partes } \\
\text { íntimas? } \\
\text { ¿Cómo se analiza un gas? } \\
\text { ¿Por qué el gas produce llama? } \\
\text { ¿Es posible que un gas contenga } \\
\text { fuego? } \\
\text { ¿Por qué el gas lacrimógeno } \\
\text { cuando choca contra el piso } \\
\text { explota? }\end{array}$ \\
\hline
\end{tabular}

Tabla No 1. Preguntas formuladas entorno al concepto "gases" por los niños y niñas de grado tercero y cuarto de Educación Básica Primaria IED. "Divino Maestro"

De los laboratorios

Las preguntas formuladas planteadas y / o resueltas permitieron:

> Construcción de hipótesis, conjeturas y predicciones referenciadas al tema, siendo la pregunta la que orientó estas acciones.

$>$ Argumentaciones pertinentes, coherentes y de mayor precisión cient́fica.

> Existió el reconocimiento de una intencionalidad definida y dara, se destaca que las preguntas elaboradas son un tanto más desde un lenguaje cotidiano, tímidamente científico.

$>$ Favorece el trabajo en equipo al reconocerse como sujetos de un lenguaje científico.

$>$ Las actitudes hacia las ciencias se fortalecen al permitirse "dudar"

> Anótese en las entrevistas no formales que al cuestionarlos sobre la importancia de las preguntas en ciencias los niños y las niñas argumentaron:

$>$ Claro son importantes porque enseñan mudho, ayudan a entender mejor lo que nos rodea.

$>$ Son importantes porque aprendemos a descubrir lo que hay detrás de ellas.

$>$ Tienen muchas ventajas como analizar e interpretar mejor lo que escribo, leo y propongo.

Formular preguntas interesantes es tener una ventaja de aprender y de soñar.

$>$ Con las preguntas, tenemos la oportunidad de evaluarnos y así saber si sabemos bien o no. 


\section{Conclusiones}

Durante la investigación se logró evidenciar una estrategia de enseñanza que apuntan y aporta a resolver uno de los problemas en que se circunsaribe la nueva didáctica de las ciencias, sus relaciones enseñanza-aprendizaje; la formulación, planteamiento y elaboración de preguntas de calidad y de carácter científico da cuenta de esto en los niveles educativos en que se adelantó el proceso investigativo.

La implementación de estas estrategias, permitieron un acercamiento "seguro" al lenguaje científico y a su actividad en razón a:

$>$ Se instaura como eje de comunicación intrapersonal e interpersonal, el primero porque establece relaciones entre lo ya conocido con los nuevos significados incorporados a su estructura conceptual y el segundo dado que es uno de los modos universales de estar con otro (Pasquall, 1990-citado por Eslava y Eslava, 2000) en cuya acción intervienen la riqueza discursiva de la disciplina, objeto de estudio en el que se involucra simultáneamente al que habla y a su interlocutor.

> Desde la hermenéutica y la heurística, la pregunta ofrece una gama de posibilidades propias para las relaciones enseñanza-aprendizaje como el lograr mantener y replicar las actitudes favorables o disposiciones hacia la ciencia misma, ayuda a iniciar procesos interactivos de aprendizaje y solución de problemas (Freire citado por Zuleta Araujo (2002) reflexiona sobre lo conocido, entre otras.

- Al planearse la acompañan los procesos del leer y el escribir dado que son vehículos apropiados para la construcción de significados o profundiza los existentes, "las ideas de la ciencia, se construyen y se aprenden expresándolas; su evolución es una condición desde el conocimiento de las formas de hablar y de escribir" (Sarda y Sanmarti, 2000).

Por tanto, es prioridad rescatar y generar espacios en donde prevalezcan las intencionalidades anteriores, de lo contrario caeríamos nuevamente en la cultura de las respuestas. Además, el saber emplear pedagógicamente las preguntas permite a niños y niñas organizar sus ideas, interpretar sus hallazgos, retomar sus ideas iniciales (Ferreira y De Longhi, 2001) siendo este tipo de estrategias y en coherencia con las opiniones de Gil Pérez y Martínez Torregrosa (1999) las que reúnen las características deseables para propuestas de enseñanza afines al trabajo científico (Ferreira y De Longhi, 2001) y posibilita comprender la complejidad del mundo, así como su actuar.

\section{Bibliografia}

Barbosa, De Carvalho, Ferreira y De Longhi, 2001. "Ejercicios de razonamiento" En tres lenguajes: enseñanza de la física en los primeros años de la Escuela Primaria" en Revista de Investigación y experiencias didácticas, Número Extra. VI Congreso Internacional sobre investigación en la didáctica de las ciencias. Pág. 139-140

Briones, G., 2004. La investigación en el aula y en la escuela Formación de docentes en investigación educativa. Módulo 2 de aprendizaje. Convenio Andrés Bello. Bogotá-Colombia.

Caldeira, H., Gomes C., Morgado, J. Y Otero, J., 2001. La formulación de preguntas como parte del control de la comprensión de la ciencia. En Enseñanza de las ciencias, Número extra, VI Congreso Internacional sobre investigación en la didáctica de las ciencias. Pág.117-118.

Chevallard, Y., 1991. La transposidión didáctica: del saber sabido al saber enseñado. Aique Grupo Editor S.A. Argentina.

Dumon, A., 1992. Formar a los estudiantes en el método experimental: ¿Utopía o problema superado? En Enseñanza de las Ciencias, 10 (1), Pág. 25-31 
Eslava de aja, L. y Eslava, E., J., 2000. La pregunta oral y escrita como factor de interacción maestro-alumno en el aula. Joumal of Science Education, Pontificia Universidad Javeriana, Vol. 1, No. 2 Periodo de Julio-Diciembre, Bogotá.

Ferreyra, A. y De Longhi, A, L, 2001. Una estrategia comunicativa coherente con modelos para "hacer ciencias" en el aula de primaria. En Enseñanza de las ciencias, Número extra, VI Congreso Internacional sobre investigación en la didáctica de las ciencias. Pág. 139-140

Gadamer, H. G., 1994. Verdad y Método. Editorial Sigueme, Salamanca, 1994, pp. 415-458.

Kuhn, T. S, 1971. La estructura de las revoluciones científicas. México. Fondo de la cultura económica.

Gil, P., Carrascosa, A. y Martínez, T. F, 1999. El surgimiento de la didáctica de las ciencias como campo espećfico de conocimientos. Revista de Educación y Cultura. Vol. 9, No. 25.

Macias, A. y Maturano, C., 2001. Lenguaje escrito en las dases de física. Revista Enseñanza de las Ciencias. Número Extra, VI Congreso Intemacional sobre investigación en la didáctica de las ciencias.

Montenegro, A, 1999. Función de la pregunta en los procesos de Razonamiento en Ciencias. Estudio Etnográfico. Bogotá: Inédito. Informe Universidad Pedagógica Nacional.

Montenegro, A, 2002 Preguntas Cognitivas y Metacognitivas en el proceso de aprendizaje, Influencia de preguntas cognitivas y metacognitivas en comprensión conceptual y en habilidad para resolver problemas en Ciencias. TEA, Revista de la Facultad de Ciencia y Tecnología. Universidad Pedagógica Nacional, No. 11. Bogotá, D.C.

Pérez, Gallego, Torres de Gallego y Gallego, 2003. Enseñanza de las ciencias experimentales y estándares aurriculares. Revista Educación y Cultura No. 63, Mes de Junio, Bogotá D.C.

Rojas, Rojas, S, P., Castellanos, M., y Vargas, E., 2002. La pregunta como estrategia didáctica en la interpretación de textos" P.F.P.D. Secretaría de Educación Distrital. Fundación Universitaria Monsenrate, Bogotá.

Rojas, Rojas, S, P., 2006. Las competencias Cognoscitivas y el concepto gases. Universidad Pedagógica Nacional. Tesis de Maestría, Departamento de Química, Facultad de Ciencia y Tecnología- Marzo de 2006.

Sarda, J., Sanmartí, N., 2000. Enseñar a argumentar científicamente: un reto de las clases de ciencias, en Revista de Investigación y experiencias didácticas, Vol. 18 (3), 405-422

Sanmarti, N., 2001. Enseñar a enseñar ciencias en secundaria: un reto muy complejo. Revista Interuniversitaria de formación de profesores. (40), Pág. 31-48.

Sutton, C., 2003. Los profesores de Ciencias como profesores del lenguaje. Revista Enseñanza de las Ciencias, Vol., 21 No. 1. Pág. 21-25

Zuleta, A, 0., 2002 La pedagogía de la pregunta. Una contribución para el aprendizaje. Revista Educación y cultura No. 59. Pág. 48-53 


\section{Anexos}

\section{Anexo No 1. Tabla No.1. Rejilla de Evaluacion Preguntas}

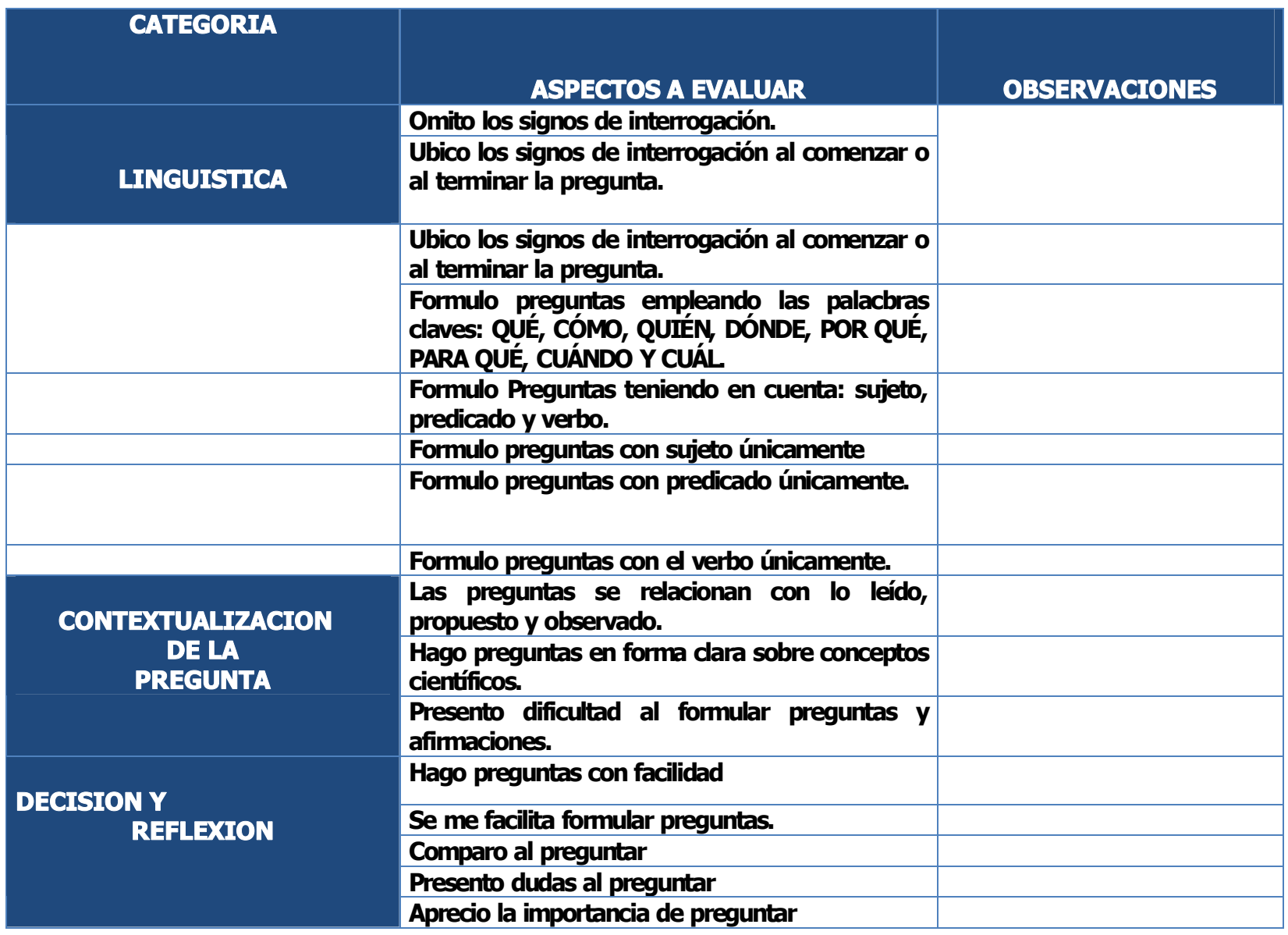

